\title{
El turismo una alternativa de desarrollo local. Potencialidades del Corredor del Santa Bárbara, Ecuador.
}

\section{Tourism an alternative local development. Potential Corridor Santa Barbara, Ecuador.}

\author{
Yonimiler Castillo Ortega ${ }^{1 *}$ y Guillermo A. Cárdenas Campoverde ${ }^{1}$ \\ ${ }^{1}$ Universidad Católica de Cuenca \\ *ycastilloo@ucacue.edu.ec
}

DOI: https://doi.org/10.26871/killkana_social.v2i4.102

\begin{abstract}
Resumen
El trabajo actual realiza una descripción de las potencialidades turísticas del Corredor del Santa Bárbara, Azuay, Ecuador. Se inicia con un debate teórico sobre el desarrollo local y el papel del turismo como fuente de generación de ingresos y empleo de algunas economías poco desarrolladas del mundo, haciéndose referencia a los elementos teóricos que abordan la sostenibilidad de la actividad turística. Se realiza un inventario de los atractivos turísticos de la zona de estudio, obteniéndose 39 manifestaciones culturales y sitios naturales que constituyen un importante potencial, al contar con varios lugares patrimonios de la nación y de la humanidad, sitios arqueológicos, gastronomía, fiestas religiosas, artesanía y técnicas milenarias como el IKAT utilizada en la elaboración de las macanas. También se complementa la oferta con varias áreas naturales protegidas y sitios con muy poca intervención humana con mucha potencialidad para el turismo de naturaleza o ecoturismo.Aunque se pudo detectar que el $59 \%$ de los atractivos, que se contabilizan, tienen alteraciones en su conservación se considera que territorio en un potencial para el desarrollo de productos culturales enriquecidos con actividades de turismo de naturaleza o ecoturismo.
\end{abstract}

Palabras clave: Turismo, Desarrollo Local, Potencialidades, Ecuador.

\begin{abstract}
The present work makes a description of the tourist potential of the Corridor of Santa Barbara, in Azuay, Ecuador. It begins with a theoretical debate on local development, and the role of tourism as an income and employment generating source for some underdeveloped world economies, it also makes reference to the theoretical elements that approach the sustainability of the tourism activity. An inventory of the tourist attractions of the area of study is conducted, yielding 39 cultural manifestations and natural sites which are an important potential, having several World and national heritages, archaeological sites, gastronomy, religious festivals, crafts and ancient techniques like IKAT used in the elaboration of Macanas. Several protected natural areas and sites with very little human intervention also complement the offer with great potential for nature tourism or ecotourism.Although it could be detected that the 59\% of the attractions, which are accounted for, have alterations in their conservation, it is considered that the territory is a potential for the development of cultural products enriched with activities of nature tourism or ecotourism.
\end{abstract}

Key words: Tourism, Local Development, Potential, Ecuador.

\section{Introducción}

El desarrollo local se ha convertido en una alternativa real a las disparidades territoriales, a la pobreza y al neoliberalismo, sobre todo en zona mucho más desfavorecidas, que han encontrado en la política de desarrollo endógeno, ventajas que mediante el aprovechamiento de las capacidades locales, han contribuido a la generación de empleo y acumulación de capital.

El turismo es una de las actividades que ha pasado a ser una importante fuente de ingreso de divisas de varias economías poco desarrolladas del mundo. De aquí la im- portancia de esta actividad como fuente de generación de ingresos y empleo para las economías locales constituyendo una alternativa real de desarrollo local.

Ecuador es un país que está apostando al turismo como alternativa de desarrollo, esto es una estrategia de gobierno actual; así lo plantea el Plan Estratégico de Desarrollo de Turismo Sostenible para Ecuador

cite[p.18]PLANDETUR2020 . En este sentido algunos territorios han emprendido acciones obteniendo experiencias positivas. 
El presente trabajo realiza un análisis de las potencialidades que tiene el territorio que comprende el Corredor del Santa Bárbara a partir de un inventario de los atractivos turísticos que este contiene. Defiende la idea de que los atractivos existentes constituyen una potencialidad para el desarrollo del turismo sostenible en la zona que comprende los municipios Gualaceo, Chordeleg y SigSig. Los resultados muestran que los 39 atractivos turísticos inventariados y jerarquizados se caracterizan por una importante riqueza cultural que predomina y natural que la complementa. Lo que convierte al territorio en un potencial para el desarrollo de productos culturales enriquecidos con actividades de turismo de naturaleza o ecoturismo.

Para el análisis se utiliza información cuantitativa y cualitativa de tipo secundaria y primaria obtenida del Ministerio de Turismo de Ecuador y del trabajo de campo del investigador. Se usaron técnicas e instrumentos como son las entrevistas, fichaje para sistematizar la información de los atractivos turísticos de la zona y la revisión documental. Para el fichaje de los atractivos turísticos se utilizó la ficha del Ministerio de Turismo del Ecuador.

\section{Tendencias teóricas sobre desarrollo local y turismo}

Las teorías sobre desarrollo local son parte de la evolución de una corriente teórica que ha tomado auge desde el siglo pasado y a la cual le han llamado las teorías del desarrollo, en las cuales se pregunta el porqué de las asimetrías entre los países y la existencia del subdesarrollo. Estas teorías tienen una base en los modelos de Solow y Swan, que a su vez tienen como antecedentes los modelos de crecimiento económicos clásicos, neoclásicos y keynesianos, entre estos el de Harrod - Domar.

En los años 80 del siglo pasado como parte de esta corriente teórica surgen las teorías del desarrollo local, originándose corrientes como desarrollo local endógeno, desarrollo local integrado, desarrollo local con un enfoque integral y desarrollo local complementario.

En el presente acápite se abordarán algunas de las tendencias actuales sobre el desarrollo local y el papel del turismo como eje de desarrollo de territorios subnacionales.

\subsection{Desarrollo local. Apuntes teóricos}

Las diferentes teorías relacionadas con el desarrollo local han surgido como alternativa de solución a las disparidades territoriales, a la pobreza y al neoliberalismo. Estas nacen a partir de las necesidades de desarrollo de las localidades más desfavorecidas (Castillo y Lazo, 2014, p.399)

Algunos autores que han realizado aportes a las teorías sobre el desarrollo local son los siguientes:

Vázquez Barquero (2000), quien se refiere al desarrollo económico local como:

“. . . un proceso de crecimiento y cambio estructural que, mediante la utilización del potencial de desarrollo existente en el territorio, conduce a elevar el bienestar de la población de una localidad o una región". (p. 21)

En esta visión sobre el desarrollo local se considera que este proceso debe realizarse desde el interior del territorio sin permitir la participación de factores exógenos como la inversión externa. Otros autores como Blacutt, M. (2013, p.128), tienen una posición diferente en la cual conciben el desarrollo local complementario como:

“. . . el proceso por el que los diferentes grupos sociales que habitan un territorio determinado en el interior de un país y viven en un régimen autonómico, conciben y ejecutan las acciones necesarias para elevar la calidad de vida de su población, acudiendo para ello al uso de sus recursos humanos y naturales, a todas sus potencialidades y a las externalidades financieras, tecnológicas y de conocimiento a las que puedan tener acceso; al mismo tiempo, convoca la voluntad de los agentes económicos, sociales, políticos, institucionales y culturales a la tarea de perfilar un cambio en las relaciones de producción que se base en la unidad de objetivos comunes planteados y ejecutados por la trilogía: EstadoEmpresa-Sociedad Civil".

En esta concepción se puede observar que las fuentes externas e internas a la localidad deben ser consideras complementarias. A esta forma de concebir el desarrollo local, Blacutt (2013) le llamó desarrollo local complementario (DELC). Este autor considera que no puede existir el desarrollo local sin la presencia del Estado, no solo en calidad de proveedor de bienes, servicios e instituciones, sino en un papel de generar acciones, asociaciones, cooperaciones y coordinaciones con los territorios locales. Tampoco puede darse sin la existencia de un proceso de descentralización que otorgue autonomías en competencias, financieras, administrativas, tecnológicas en las áreas geográficas locales.

Las teorías neoclásicas del desarrollo regional asumen que en la región hay pleno empleo, competencia perfecta, por lo tanto, habrá un solo bien homogéneo, no habrá costos de transporte. También se supone que todas las regiones, independientemente de sus características geográficas y dotaciones de recursos tienen la misma función de producción, función que supone rendimientos constantes de escala. Por otra parte, asume que cada factor tiene rendimientos marginales, por lo tanto, retornos decrecientes; así, cada uno de los factores de producción, tal como el trabajo y el capital, reciben sus retornos en función de la cantidad con relación al otro. No existirá progreso técnico y la oferta de trabajo será constante (Blacutt, 2013, p.122)

También la tecnología juega un papel importante en las respuestas al crecimiento a largo plazo que tuvieron, en la década de 1950 y 1960, los países industriales.

Luego en el modelo neoclásico la retribución de los factores agota el producto final. No quedan recursos para asignar a $\mathrm{I}+\mathrm{D}$, puesto que la tecnología es un bien público (en el sentido de un bien no rival y no excluible). Posterior el aumento de la productividad ha de ser necesariamente 
exógeno(en el sentido de que el progreso tecnológico no es explicado por el propio modelo).

Toda la teoría neoclásica contrasta con los modelos de crecimiento endógeno unisectorial ya que el supuesto de crecimiento tecnológico exógeno es intelectualmente insatisfactorio, convirtiéndose en un modelo de crecimiento en el que lo único que no se explica es el crecimiento. Por otro lado está la no explicación de la convergencia de rentas a nivel espacial.

La Comisión Económica para América Latina (Vázquez, 2000) plantea que el desarrollo económico local es un proceso de crecimiento y cambio estructural de la economía de una ciudad, comarca o región, en el que se pueden identificar al menos, tres dimensiones (Coffey, W. J. and Polese, M. , 1985; Stöhr, 1985): una económica, caracterizada por un sistema de producción que permite a los empresarios locales usar, eficientemente, los factores productivos, generar economías de escala y aumentar la productividad a niveles que permiten mejorar la competitividad en los mercados; otra sociocultural, en que el sistema de relaciones económicas y sociales, las instituciones locales y los valores sirven de base al proceso de desarrollo; y otra, política y administrativa, en que las iniciativas locales crean un entorno local favorable a la producción e impulsan el desarrollo sostenible.

Una de la teorías sobre el desarrollo local que también ha tomado auge en los últimos veinte años es la del desarrollo endógeno. Según Vazquez (2007) “. . . desde los inicios de los años ochenta aparece la teoría del «desarrollo endógeno»". Esta interpretación surge de la confluencia de dos líneas de investigación: una, que nace como consecuencia del intento de encontrar una noción de desarrollo que permitiera actuar para lograr el desarrollo de localidades y territorios retrasados (Friedmann, 1978; Stöhr, 1981); y otra, que aparece como consecuencia del análisis de los procesos de desarrollo industrial endógeno en localidades y regiones del sur de Europa (Becattini, 1979; Brusco, 1982; Fuà, 1983; Garofoli, 1983)(Vázquez, 1983, p.186).

Según Vázquez Barquero (2000) en la década de los noventa la disminución del Estado obligó la intervención de actores locales y regionales, y junto con esta se empezó a estructurar una estrategia de descentralización que abriera paso a las iniciativas locales, y una perspectiva de desarrollo con enfoque territorial acompañada del cambio paradigmático del desarrollo exógeno por el endógeno. . . tomado de (Rosas y Lara, 2013, p.63). El mismo autor Vazquez (2007) plantea que el desarrollo endógeno se trata de una aproximación territorial al desarrollo, que hace referencia a los procesos de crecimiento y acumulación de capital de una localidad o un territorio, que tiene cultura e instituciones que le son propias y en las que se basan las decisiones de ahorro e inversión (p.184)

En la actualidad existe un debate importante sobre la llamada nueva geografía económica y la economía regional. La cual tiene su base en la "nuevas" teorías del comercio internacional, presentadas por Helpman y Krugman (1985); que a su vez fueron posibles gracias al modelo de competencia monopolística desarrollado por Dixit y Stiglitz (1977).

Esta visión de la economía, que considera el espacio y el tiempo en sus análisis, aporta un enfoque del desarrollo territorial basado en los rendimientos creciente y la competencia imperfecta, lo cual explica los desequilibrios territoriales. Helpman y Krugman (1985) utilizan modelos en que los factores son totalmente móviles y para transportar los bienes se incurrirían en costes de transportes. Intenta demostrar dos postulados: Uno, los rendimientos crecientes tienen, de hecho, una influencia permanente en la economía, pudiéndose percibir en los procesos acumulativos, resultando las ciudades. Dos, los acontecimientos históricos han jugado un papel decisivo en la distribución geográfica de la producción, explicándose de esta manera el desarrollo divergente de las regiones.

Helpman y Krugman realizan un modelo para explicar la concentración geográfica industrial (sistema centro periferia), y según el modelo, la concentración nace, básicamente, de la interacción de los rendimientos crecientes, los costes de transporte y la demanda. La explicación se basa en la existencia de dos fuerzas centrípetas, que tienden a conservar la existencia del centro industrial y una fuerza centrífuga, que tiende a destruirlo.

Dentro de las fuerzas centrípetas se pueden mencionar:

- El deseo de las empresas de situarse cerca del mercado más grande.

- El deseo de los trabajadores de poder acceder a más bienes (producidos por otros trabajadores)

La fuerza centrífuga es el incentivo de las empresas de trasladarse para abastecer el mercado agrícola de la periferia.

Las últimas tendencias relacionadas con el desarrollo local incorporan los elementos del medio ambiente en las concepciones y estrategias de desarrollo local. El deterioro de los componentes de la naturaleza ha comenzado a ser limitantes importantes a los procesos de desarrollo local.

En Italia, este camino ha llevado a la "Compañía de los territorialistas" para desarrollar modelos funcionales de planificación en la búsqueda de un desarrollo local autosostenible (Mancini, 2014, p.44). Según este autor el prefijo "auto" hace hincapié en la necesidad de que el modelo asegure la capacidad de autoreproducción local de los recursos empleados en el proceso de puesta en valor de yacimientos patrimoniales sin necesidad de apoyo externo.

Según Castillo y Lazo (2014) también se han introducido importantes conceptos en la nueva visión del desarrollo local, como lo son: las cadenas productivas, los cluster, el emprendimiento, la gestión del conocimiento, el capital humano y la innovación. En este sentido se encuentran algunos trabajos que se centran en el estudio de las ciudades o regiones inteligentes o basadas en el conocimiento (Rodríguez, 2014); como son: la generación de economías intensivas en conocimiento (Derudder y Witlox, 
2016), y otros en la cultura local como factor estratégico de planificación urbana y regional (Fachinelli y et al., 2014)

En consideración del autor en la actualidad y realidad del Ecuador es necesario adoptar aspectos de la concepción de desarrollo endógeno de Vázquez Barquero, ya que los procesos de descentralización del país lo permiten. Pero a la vez se debe incorporar elementos como la innovación y los encadenamientos productivos como formas de estimular el crecimiento económico inclusivo desde el territorio y con el aprovechamiento de recursos externos como las remesas que son muy importantes en algunas localidades.

\subsection{Turismo. Concepciones teóricas}

El turismo es un fenómeno social, cultural y económico, relacionado con el movimiento de las personas a lugares que se encuentran fuera de su residencia habitual por motivos personales o de negocios/profesionales, tiene que ver con sus actividades, de las cuales algunas implican un gasto turístico (OMT, 2010).

El art 2 de la Ley de Turismo del Ecuador (2002) (última modificación diciembre de 2014) define al turismo como "el ejercicio de todas las actividades asociadas con el desplazamiento de personas hacia lugares distintos al de su residencia habitual, sin ánimo de radicarse permanentemente en ellos".

En la década de 1990 se hace un análisis de la conceptualización del turismo sobre la base del concepto de desarrollo sostenible que se emitió en el informe de la Conferencia de las Naciones Unidas para el Medio Ambiente y Desarrollo (CNUMAD), realizada en Río de Janeiro en 1992.

La primera definición a la que se hará referencia es a la que emite la OMT en el año 1999 donde plantea que el Turismo Sostenible: es un modelo de desarrollo económico concebido para mejorar la calidad de vida de la comunidad receptora y facilitar al visitante una experiencia de alta calidad del medio ambiente del que tanto la comunidad anfitriona como los visitantes dependen.

En relación a esto Hunter y Green (1995), plantean que el turismo sostenible debe de perseguir tres objetivos a corto y largo plazo:

- Conciliar las necesidades y deseos de la comunidad que sirve de destino en términos de elevar los estándares y la calidad de vida. (CRITERIO SOCIAL DE SOSTENIBILIDAD).

- $\quad$ Satisfacer las demandas de los turistas, y de la industria turística, y continuar atrayéndolos para alcanzar la primera meta (CRITERIO ECONÓMICO).

- Proteger los recursos naturales que son base para el turismo, en armonía con los componentes naturales, construidos y culturales (CRITERIO NATURAL).

Rivas (1997) plantea un cuarto objetivo que complementa lo señalado por Hunter y Green es que el turismo sostenible debe proteger la identidad cultural de la comunidad destino, a través de asegurar una relación sana entre la comunidad local y el/la turista. (RELACIONADO CON EL
ASPECTO CULTURAL) Por lo que el turismo sostenible se entiende por un turismo diferente caracterizado por evitar las consecuencias indeseables tanto en el plano económico, como social y natural presuponiendo una nueva ética del turismo que permita ser productivo (desde lo económico), responsable (desde lo social) y consciente (en lo natural). Además se amplía el horizonte de desarrollo temporal al largo plazo, satisfaciendo las necesidades de las generaciones presentes y futuras.

En este sentido el Comité de Desarrollo Sostenible de la OMT en su reunión de Tailandia, en marzo de 2004, acordó examinar las definiciones anteriores sobre turismo sostenible. Emitiéndose una nueva definición sobre el desarrollo sostenible del turismo, divulgada en el documento Desarrollo Sostenible del Turismo, en agosto de 2004. Planteándose lo siguiente:

"Las directrices para el desarrollo sostenible del turismo y las prácticas sostenibles son aplicables a todas las formas de turismo en todos los destinos, incluidos el turismo de masa y los diversos segmentos turísticos. Los principios de sostenibilidad se refieren a los aspectos ambiental, económico y sociocultural del desarrollo turístico, habiéndose de establecer un equilibrio adecuado entre estas tres dimensiones para garantizar su sostenibilidad a largo plazo.

El turismo sostenible por tanto debe:

Dar un uso óptimo a los recursos ambientales que son un elemento fundamental del desarrollo turístico, manteniendo los procesos ecológicos esenciales y ayudando a conservar los recursos naturales y la diversidad biológica.

Respetar la autenticidad sociocultural de las comunidades anfitrionas, conservar sus activos culturales, arquitectónicos y vivos sus valores tradicionales, y contribuir al entendimiento y a la tolerancia intercultural. Asegurar unas actividades económicas viables a largo plazo, que reporten a todos los agentes unos beneficios socioeconómicos bien distribuidos, entre los que se cuente oportunidades de empleo estable y de obtención de ingresos y servicios sociales para las comunidades anfitrionas, y que contribuya a la reducción de la pobreza.çitado por (Roldán, 2012, p.134)

Como se puede observar no hay una definición clara en relación a este tema, por lo que Miguel Ángel Acerenza en su artículo titulado: "Sostenibilidad del turismo III: Confusión sobre la definición del desarrollo sostenible del turismo" (Acerenza, 2011, p.20) publicado en marzo del 2011, expone una nueva definición en este sentido y plantea lo siguiente:

El desarrollo del turismo sostenible debe ser:

"el que satisface las necesidades de los turistas actuales, respetando los principios de sostenibilidad económica, sociocultural y ambiental, así como la diversidad biológica y los ecosistemas de los cuales depende, para no comprometer la capacidad de las futuras generaciones para satisfacer sus propias necesidades en materia turística". 
El turismo es una industria que ha pasado a ser la principal fuente de ingreso de divisas de las 48 economías menos desarrolladas del mundo (excluida la exportación de petróleo), el generador de 45 de las exportaciones de servicios de los países en desarrollo y la principal exportación en 80 de estos casos (Brida, London, y Rojas, 2014, p.60). De aquí la importancia de esta actividad como fuente de generación de ingresos y empleo para las economías locales constituyendo una alternativa real de desarrollo local. El turismo posee las características de ser una industria con mano de obra intensiva y poseer un gran número de conectividades con otras industrias, así como también generar importantes efectos de spillover entre productores y consumidores (Brida y cols., 2014, p.60).

Ecuador es un país que está apostando al turismo como alternativa de desarrollo, esto es una estrategia de gobierno actual; así lo plantea el Plan Estratégico de Desarrollo de Turismo Sostenible para Ecuador (Asamblea Constituyente, 2007, p.18).

En el período 2006-2014 se puede percibir un incremento en la actividad turística en Ecuador, lo cual se muestra en el crecimiento sostenido de las entradas de extranjeros al Ecuador como se puede observar en la Figura 1

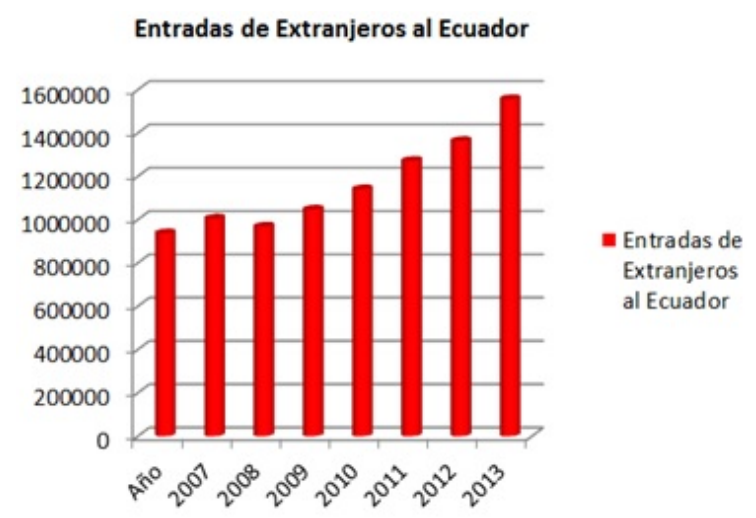

Figura 1. Entradas de Extranjeros al Ecuador período 2006-2014 Fuente: Boletín Estadísticas Turísticas 2006-2014. Elaboración Propia

Existen varias experiencias de desarrollo turístico local en el país, dentro de estas se puede mencionar: la Comunidad Aguas Blancas ubicada en el Parque Nacional Machalilla, RUNA TUPARI - OTAVALO, Baños de Ambato entre otros.

En la presente investigación se estudia el caso del Corredor Turístico del Santa Bárbara, se expone las potencialidades que el territorio tiene para impulsar el turismo como alternativa de desarrollo local.

\section{Metodologías y técnicas}

Durante el desarrollo de la presente investigación se utilizaron los métodos teóricos como el analítico y el sintético que permitieron realizar un análisis de las principales corrientes sobre el desarrollo local y turismo, así como algunos postulados actuales sobre distintas características de la zona de estudio, la cuantificación de los recursos turísticos y el levantamiento de la información que fundamenta los resultados y las conclusiones.

Se usaron técnicas e instrumentos que permitieron la obtención de información primaria y secundaria como son:

La entrevista a técnicos de turismo de los diferentes municipios y a pobladores de la zona donde se encuentran los atractivos turísticos junto a la revisión de documentos del Ministerio de Turismo permitió la obtención de información histórica y relevante sobre los mismos.

La información obtenida sobre los atractivos turísticos se sistematizó utilizándose la ficha del Ministerio de Turismo del Ecuador.

La fotografía de los atractivos turísticos permitió la descripción de algunas características que fueron visualizadas por el investigador.

De la revisión del catastro turístico de los municipios se obtuvo la información sobre la cantidad de empresas turísticas que se dedican a prestar los distintos servicios de alojamiento, alimentación, transportación y operación turística. Así mismo de la revisión del documento del Plan de Desarrollo y Ordenamiento Territorial se adquirieron datos generales del territorio.

El tipo de investigación que se aplicó es el estudio de caso cualitativo con un alcance descriptivo ya que lo que se obtiene como resultados es una descripción de las características y potencialidades turísticas que tiene el Corredor del Santa Bárbara.

\section{Resultados}

En el presente acápite se exponen los resultados obtenidos de la aplicación de los instrumentos de la investigación en la zona de estudio. Para esto se iniciará con una explicación del territorio que comprende el Corredor del Santa Bárbara.

\subsection{Zona de estudio Corredor del Santa Bárbara}

El Corredor Turístico del Santa Bárbara se localiza al sureste de la provincia del Azuay, ubicado a $35 \mathrm{~km}$ del cantón Cuenca en la subcuenca del río Santa Bárbara, perteneciente a la cuenca hidrográfica del río Paute; a una altura de 2.370 metros sobre el nivel del mar, con una temperatura promedio de $14^{\circ}$ a $16^{\circ} \mathrm{C}$ y una extensión de $952 \mathrm{~km}^{2}$.

El Santa Bárbara está compuesto por los cantones de Gualaceo, Chordeleg y Sigsig (ver Fig 2), tiene 82.196 habitantes entre hombres y mujeres. Cuenta con 19 parroquias, llenas de encanto, cultura y tradición, tierra de leyendas, mitología e historia Cañarí. Es una región que posee recursos naturales, arqueológicos, arquitectónicos, convirtiéndola en un centro histórico, con una estructura física proveniente del pasado.

\footnotetext{
${ }^{1}$ Censo de población y vivienda del Ecuador, 2010
} 


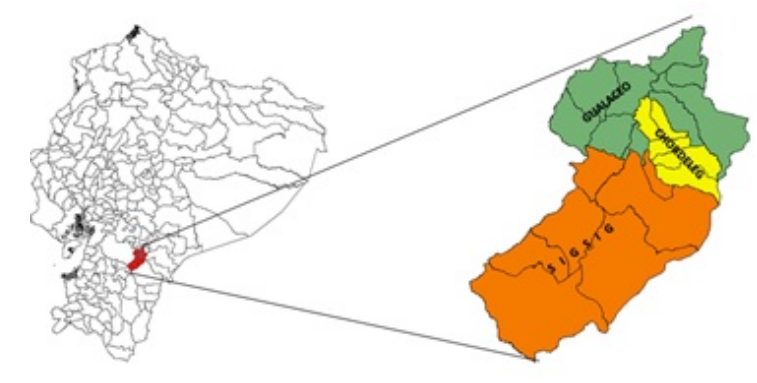

Figura 2. Mapa del Corredor del Santa Bárbara Fuente: Elaboración Propia

Sus ríos principales son el Santa Bárbara y el San Francisco. Cabe indicar que es una zona muy rica en tradición arqueológica y folklórica.

El corredor se caracteriza por sus famosos tejidos de las tradicionales chompas que son elaboradas en rústicos talleres y por el bordado que se utiliza en blusas, polleras, manteles y chales que se las puede apreciar en las propias cholas gualaceñas como parte de su atuendo, constituyen un imán para los turistas.

Los centros artesanales instalados al servicio del visitante, donde se puede apreciar de cerca las manufacturas de hombres y mujeres dedicados al tejido de chales, bufandas, cinturones y las famosas "macanas" que en quichua significa especie de rebozos, utilizando para su elaboración la milenaria técnica del "ikat". Todos ellos confeccionados en vivos colores y hermosos diseños.

Gualaceo, uno de los cantones que lo componen es Patrimonio Cultura de la Nación, fue nombrado "El Jardín Azuayo" por artistas y poetas, pues es próspero en un entorno de paz, gracias a su gente hospitalaria, su clima favorable y naturaleza privilegiada. Contando con sitios naturales como: "Las Lagunas de Maylas, el bosque protector de Collay, bosque protector de Aguarongo, etc.

El cantón de Chordeleg encontramos San Martín de Puzhio, donde tenemos el chorro de Tasqui, "en el que Los recursos agua, bosque y suelo, hacen de Tasqui una de las principales fuentes de abastecimiento de agua para Chordeleg y varias comunidades y se puede apreciar la belleza paisajística que aún esta zona conserva, así como; el infiernillo que es un cañón que fue esculpido por la fuerza invencible de la naturaleza. Los lados de este cañón dan la apariencia de unirse en la cima formando un túnel por el cual pasan las aguas de este histórico río, mientras hace este recorrido usted disfrutará de Cascadas, Cuevas, el cantar de los pájaros, típicas casas andinas, vistas únicas del Volcán Fasayñan, Cruz de Misión y otro de sus principales recursos naturales como es Las Tres Lagunas en las que desde las rocas de las montañas se origina una cascada.

También se encuentra la Candonga más grande del mundo, la cual es motivo de visitas de muchos turistas.

El Cantón Sigsig también cuenta con atractivos importantes, por ejemplo el centro cantonal fue declarado Patrimonio Cultural de la Nación en el año 2002; están las famosas Cuevas de Chobshi, "La historia cuenta que Chobshi es un vestigio que data 10000 años A.C. y el principal componente de su estructura es la piedra. El complejo arqueológico de Sigsig está integrado además por la Cueva Negra, las ruinas de Shabalula, las lagunas de Nari y Quingos, el Castillo del Inca. También cuenta con una importante riqueza cultural, llena de tradiciones y manifestaciones propias, expresadas en el arte y la artesanía, la música, la danza, la gastronomía, los sabores ancestrales; la historia oral del origen de las guacamayas, y la serpiente, los cerros y lagunas sagradas, los antepasados honrosos de los caciques cañaris, las alianzas con el incario y los vestigios de su presencia, el funcionamiento de la Comuna San Sebastián y su particular organización que aún persiste, el pasado colonial y la presencia de una religiosidad ferviente.

La principal fuente económica del corredor del santa barbará son sus recursos naturales, agricultura, dentro de esta sus habitantes se dedican al cultivo de verduras, legumbres, maíz, caña de azúcar, frutas, etc. Aunque el turismo es una alternativa importante, todavía no es la principal fuente de ingresos.

\subsection{Potencialidades turísticas del Corredor del Santa Bárbara}

Es importante exponer tres conceptos claves que se utilizaron en la clasificación de los atractivos turísticos del Corredor del Santa Bárbara, estos son: atractivos turísticos, inventario de atractivos turísticos y jerarquización de atractivos turísticos.

El atractivo turístico se define como el conjunto de elementos materiales y/o inmateriales que son susceptibles de ser transformados en un producto turístico que tenga capacidad para incidir sobre el proceso de decisión del turista provocando su visita a través de flujos de desplazamientos desde su lugar de residencia habitual hacia un determinado territorio. Este último se transforma de esta manera en un destino turístico. (Organización Mundial de Turismo, 2004). En la presente investigación, los atractivos turísticos del Corredor del Santa Bárbara clasificarán en dos grandes grupos: Sitios Turísticos Naturales y Manifestaciones Culturales según plantea la metodología de inventarios turísticos del Ministerio de Turismo del Ecuador (MINTUR).

Los sitios turísticos naturales son todos los elementos de la naturaleza con determinada atracción que motiva el sujeto a dejar su lugar de origen por un determinado tiempo, con la finalidad de satisfacer necesidades de recreación y esparcimiento como lo son: los bosques, playas, lagos, lagunas, entre otros.(Hernández, 1991)

Las manifestaciones culturales según (Alfaro, 2007), están constituidas por todos aquellos elementos y manifestaciones tangibles o intangibles producidas por las sociedades, resultado de un proceso histórico en donde la reproducción de las ideas y del material se constituyen en factores que identifican y diferencian a ese país o región.

El inventario de los atractivos turísticos es el proceso mediante el cual se registra ordenadamente los factores 
físicos, biológicos y culturales que como conjunto de atractivos, efectiva o potencialmente puestos en el mercado, contribuyen a conformar la oferta turística del país. Proporcionan información importante para el desarrollo del turismo, su tecnificación, evaluación y zonificación en el sentido de diversificar las áreas de desarrollo turístico (MINTUR).

Según López O (1998), la jerarquización de los recursos turísticos, representa una primera aproximación a la valoración que poseen estos, desde el punto de vista de su atracción.

En el PLANDETUR 2020 de Ecuador se manifiesta que, para cada uno de los atractivos turísticos existe una jerarquización con el siguiente significado:

JERARQUÍA IV.Atractivo excepcional de gran significación para el mercado turístico internacional, capas de motivar por si solo una importante corriente de visitantes actual o potencial.

JERARQUÍA III.Atractivo con rasgos excepcionales en un país, capas de motivar una corriente actual o potencial de visitantes del mercado interno, y receptivo, en menor porcentaje al internacional, ya sea por si solo o en conjunto con otros atractivos contiguos.

JERARQUÍA II.Atractivo con algún rasgo llamativo, capaz de interesar a visitantes de larga distancia, ya sea de mercado interno, y receptivo, que hubiesen llegado a la zona por otras motivaciones turísticas, o de motivar corrientes turísticas actuales o potenciales, y atraer al turismo fronterizo de esparcimiento.

JERARQUÍA I.Atractivos sin méritos suficientes para considerarlos a nivel de las jerarquías anteriores, pero que igualmente forman parte del patrimonio turístico como elementos que pueden complementar a otros de mayor jerarquía en el desarrollo y funcionamiento de cualquiera de las unidades que integran el espacio turístico.

Para determinar las potencialidades turísticas del Corredor del Santa Bárbara se realizó un inventario de los atractivos turísticos de la zona. La información fue obtenida del Ministerio de Turismo coordinación zonal 6 del Ecuador y del levantamiento in situ del investigador. En la tabla 1 , se expone un listado con todos los atractivos turísticos de la zona:

Para que se perciba la riqueza y el potencial turístico con que cuenta esta zona objeto de estudio se expone a continuación una descripción de los principales atractivos turísticos según la jerarquía de estos:

\begin{tabular}{|c|l|}
\hline Nombre del Atractivo & \multicolumn{1}{c|}{ Breve descripción } \\
\hline Artesanías de Bullcay-Bullzhun & \multicolumn{1}{|c|}{$\begin{array}{l}\text { Sus principales productos son: Las macanas, chales, } \\
\text { bufandas y cinturones, con sus colores y diseños de } \\
\text { alta calidad con reconocimientos a nivel nacional e } \\
\text { internacional. } \\
\text { La técnica utilizada por más de quinientos años por } \\
\text { hombres y mujeres es la llamada técnica del IKAT. }\end{array}$} \\
\hline
\end{tabular}

${ }^{2}$ Plan Estratégico de Desarrollo de Turismo Sostenible para Ecuador
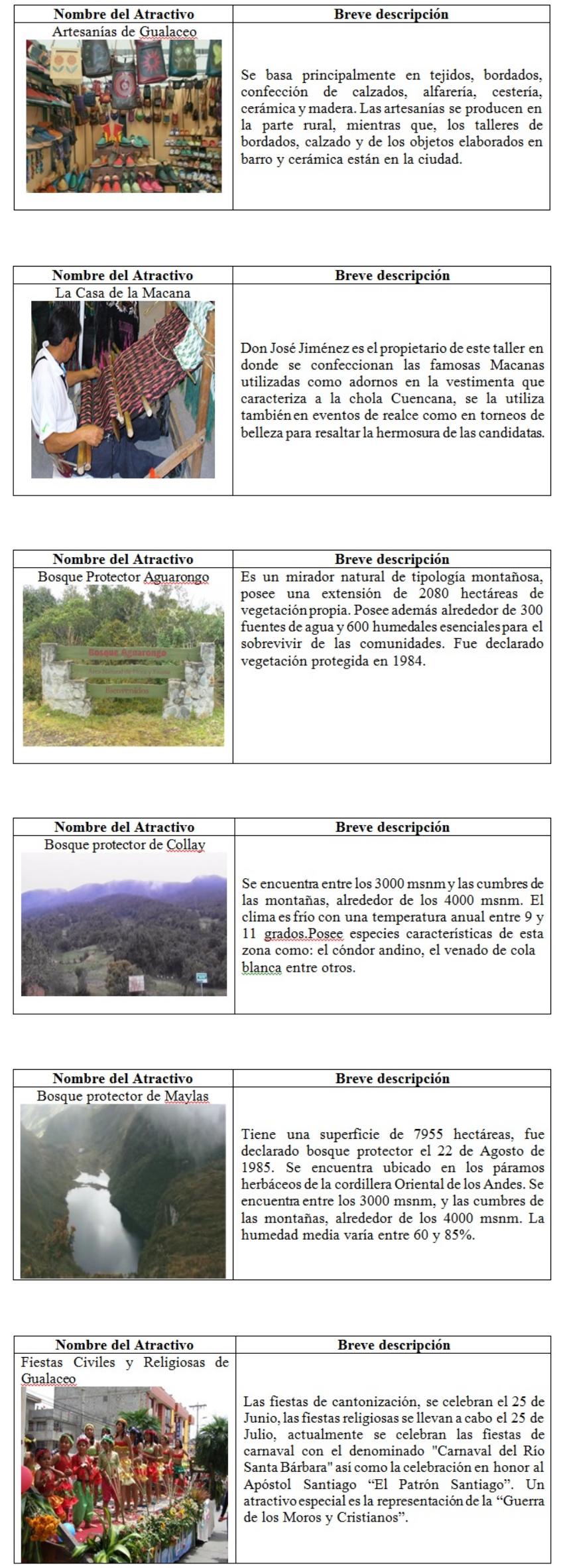
Tabla 1 Listado de Atractivos Turísticos Corredor del Santa Bárbara

\begin{tabular}{|c|c|c|c|c|c|c|}
\hline Nombre del Atractivo & Categoría & Tipo & Subtipo & Jerarquía & Estado de Conservación & Difusión del Atractivo \\
\hline Artesanías de Bullcay-Bullzhun & Cultural & Etnográfico & Artesanías & 2 & Alterado & Local, Provincial \\
\hline Artesanías de Gualaceo & Cultural & Etnográfico & Artesanías & 2 & Alterado & Local, Provincial \\
\hline La Casa de la Macana & Cultural & Etnográfico & Artesanías & 2 & Alterado & Local, Provincial \\
\hline Mercado Santiago de Gualaceo & Cultural & Etnográfico & Artesanías & 2 & Conservado & Local, Provincial \\
\hline Alfarería & Cultural & Etnográfico & Artesanías & 1 & Alterado & Local, Provincial \\
\hline Teje Mujeres & Cultural & Etnográfico & Artesanías & 1 & Alterado & Local, Provincial \\
\hline Bosque Protector Aguarongo & Natural & Bosque Protector & Colina & 1 & Alterado & Local, Provincial \\
\hline Bosque protector de Collay & Natural & Bosque Protector & Colina & 1 & Alterado & Local, Provincial y Nacional \\
\hline Bosque protector de Maylas & Natural & Caminos Pintorescos & Senderos & 1 & Alterado & Local, Provincial y Nacional \\
\hline Centro Artesanal Gualaceo & Cultural & Etnográfico & Artesanías & 1 & Alterado & Local, Provincial y Nacional \\
\hline Centro Histórico de Gualaceo & Cultural & Histórico & Arquitectura Civil & 2 & Alterado & Local, Provincial y Nacional \\
\hline Comunidad de Caguashun & Cultural & Etnográfico & Grupos Étnicos & 1 & Alterado & Local, Provincial \\
\hline Fiestas Civiles y Religiosas de Gualaceo & Cultural & Acontecimientos & Fiestas & 2 & Alterado & Local, Provincial y Nacional \\
\hline Gastronomía de Gualaceo & Cultural & Etnográfico & Comidas Típicas & 3 & Conservado & Local, Provincial y Nacional \\
\hline Orquidiario Ecuagenera & Cultural & Realizaciones Técnicas & Jardín & 3 & Conservado & Local, Provincial y Nacional \\
\hline Mirador el Calvario & Cultural & Histórico & Religioso & 3 & Conservado & Local, Provincial y Nacional \\
\hline Comunidad de San Juan y Festival de la Siembra & Cultural & Etnográfico & Grupos Étnicos & 2 & Conservado & Local, Provincial \\
\hline $\begin{array}{l}\text { Riveras de la unión de los } \\
\text { ríos Santa Bárbara-San Francisco }\end{array}$ & Natural & Ríos & Riveras & 2 & Alterado & Local, Provincial y Nacional \\
\hline Parroquia de Jadán & Natural & Ríos & Riveras & 2 & Alterado & Local, Provincial y Nacional \\
\hline Arcos de Identidad del Sigsig & Cultural & Histórico & Arquitectura Civil & 2 & Conservado & Local, Provincial y Nacional \\
\hline Centro Histórico de Sigsig & Cultural & Histórico & Arquitectura Civil & 1 & Alterado & Local, Provincial y Nacional \\
\hline Cerro Fasayñan & Natural & Montaña & Colina & 2 & Conservado & Local, Provincial y Nacional \\
\hline Fiestas Civiles y Religiosas Chordeleg & Cultural & Acontecimientos & Fiestas & 1 & Alterado & Local, Provincial y Nacional \\
\hline Bordados en Tela & Cultural & Etnográfico & Artesanías & 2 & Alterado & Local, Provincial y Nacional \\
\hline Hornado de Sombreros & Cultural & Etnográfico & Artesanías & 2 & Alterado & Local, Provincial y Nacional \\
\hline Paja Toquilla & Cultural & Etnográfico & Artesanías & 2 & Conservado & Local, Provincial y Nacional \\
\hline Esculturas en Madera & Cultural & Etnográfico & Artesanías & 1 & Alterado & Local, Provincial y Nacional \\
\hline Guitarras de San Bartolomé & Cultural & Etnográfico & Artesanías & 2 & Conservado & Local, Provincial y Nacional \\
\hline Parroquia San Bartolomé & Cultural & Etnográfico & Grupos Étnicos & 1 & En proceso de deterioro & Local, Provincial y Nacional \\
\hline Parroquia Jima & Cultural & Etnográfico & Grupos Étnicos & 1 & Conservado & Local, Provincial y Nacional \\
\hline Playas de Shingate & Natural & Ríos & Riveras y remansos & 1 & Alterado & Local, Provincial y Nacional \\
\hline Sitio Arqueológico de Chobshi Shabalula & Cultural & Zona Histórica & Sitio Arqueológico & 1 & En proceso de deterioro & Local, Provincial y Nacional \\
\hline Artesanías de Chordeleg & Cultural & Etnográfico & Artesanías & 2 & Conservado & Local, Provincial \\
\hline Museo de Chordeleg & Cultural & Etnográfico & Artesanías & 2 & Conservado & Local, Provincial \\
\hline Centro Histórico de Chordeleg & Cultural & Etnográfico & Artesanías & 1 & Alterado & Local, Provincial \\
\hline Fiestas Civiles y Religiosas Chordeleg & Cultural & Acontecimientos & Fiestas & 1 & Conservado & Local, Provincial \\
\hline Parroquia Principal & Cultural & Etnográfico & Grupos Étnicos & 1 & Alterado & Local, Provincial \\
\hline Sitio Arqueológico de Llaber & Cultural & Histórico & Sitio Arqueológico & 1 & Alterado & Local, Provincial \\
\hline Tres lagunas de Principal & Natural & Ambientes Lacustres & Lagunas & 2 & Conservado & Local, Provincial \\
\hline
\end{tabular}
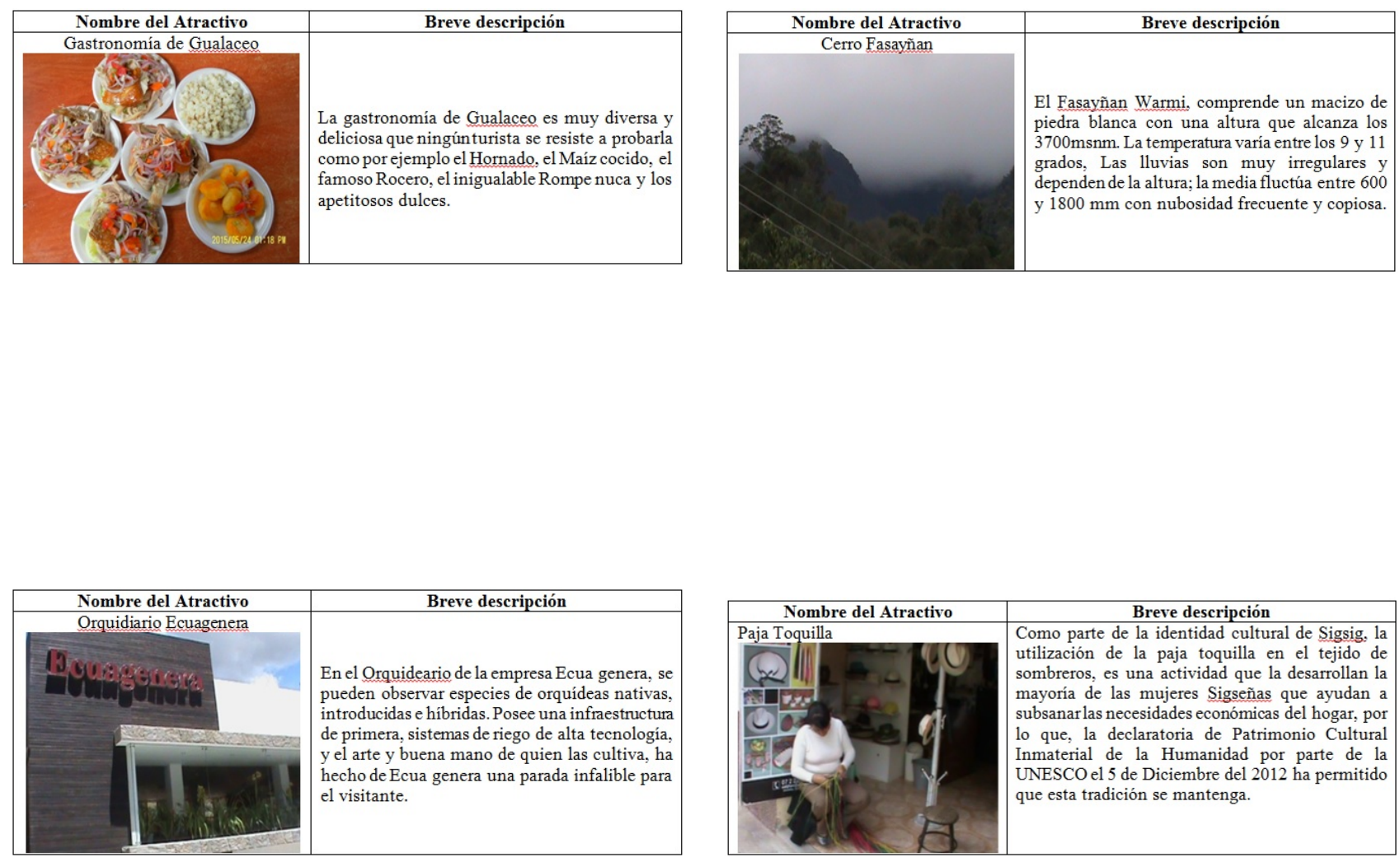


\begin{tabular}{|l|l|}
\hline \multicolumn{1}{|c|}{ Nombre del Atractivo } & \multicolumn{1}{c|}{ Breve descripción } \\
\hline Guitarras de San Bartolomé & $\begin{array}{l}\text { La fabricación de las guitarras requiere de gran } \\
\text { habilidad y paciencia, dones que los moradores de } \\
\text { San Bartolomé poseen. Varían en precio y calidad, } \\
\text { cada una está formada en sus distintas partes por } \\
\text { diferentes tipos de madera como palo de rosa, pino, } \\
\text { caoba, capuli, garantizando excelente sonido y } \\
\text { durabilidad. }\end{array}$ \\
\hline
\end{tabular}

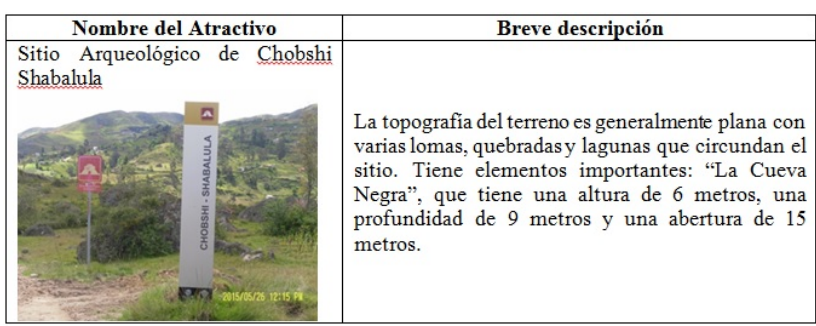

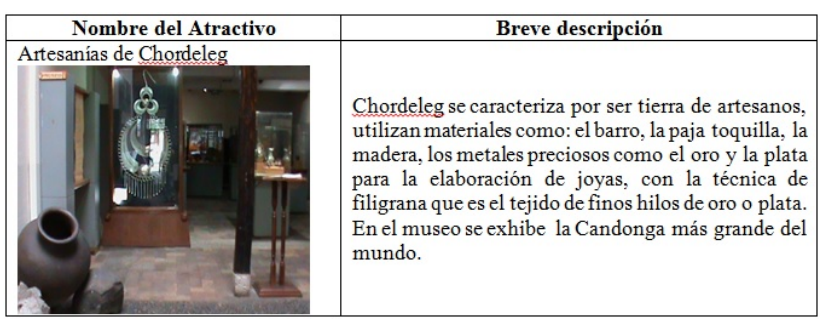

\begin{tabular}{|c|c|}
\hline Nombre del Atractivo & \multicolumn{1}{|c|}{ Breve descripción } \\
\hline Sitio Arqueológico de Llaber & \\
& $\begin{array}{l}\text { El sitio está emplazado en un entorno natural y } \\
\text { paisajístico, bañado por los río Santa Bárbara y } \\
\text { Punguhuaycu, resguardado por montañas y valles } \\
\text { que albergan ecosistemas naturales como el páramo } \\
\text { y bosques, dondela flora y fauna presenta un buen } \\
\text { estado de conservación. }\end{array}$ \\
\hline
\end{tabular}

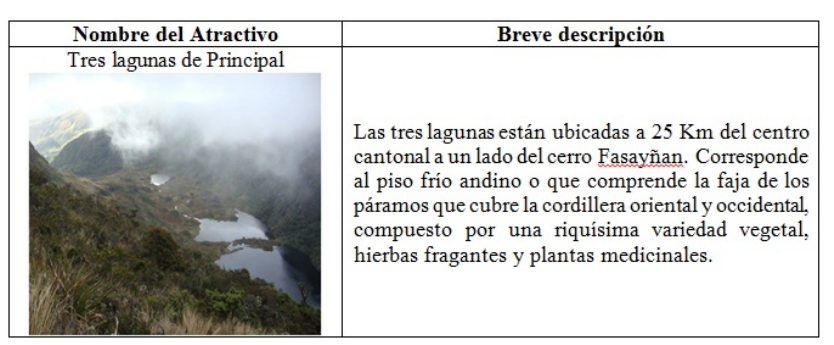

Como se puede observar se han inventariado 39 atractivos turísticos, de los cuales unos son más visitados que otros a partir de la jerarquía que se les ha otorgado a estos.

El $67 \%$ de los atractivos son culturales lo que les da un predominio a estos según se muestra en la Fig. 3. La artesanía en diferentes materiales (metales, cuero, hilo, paja toquilla, barro), las técnicas antiguas utilizadas en el proceso de producción de estas, la gastronomía autóctona, las festividades y actividades religiosas dotan al territorio de un importante potencial cultural sobre el cual debe girar el producto turístico principal, el cual deberá estar enfocado en la modalidad de turismo cultural.

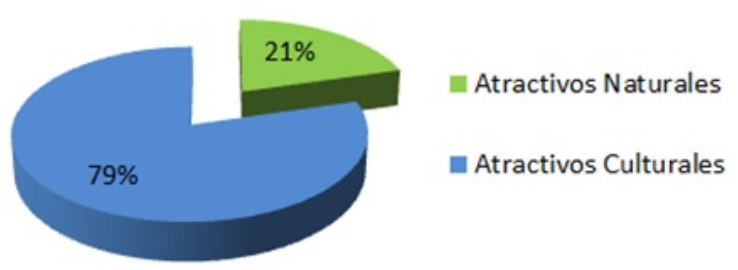

Figura 3. Distribución de los Atractivos del Corredor del Santa Bárbara por Categorías Fuente:Tabla No.1. Elaboración Propia

También el territorio tiene potencialidades para el turismo de naturaleza o ecoturismo ya que cuenta con tres áreas naturales protegidas y otras con muy poca intervención del hombre como lo son tres lagunas y el cerro fasayñan. Además si se le adiciona la existencia de atractivos históricos como lo son los dos sitios arqueológicos con que cuenta la zona, esto hace que estemos frente a un territorio rico en cultura, historia y naturaleza lo que hace que el diseño de productos turísticos integrales donde se combinen las tres opciones sea una importante opción para el desarrollo local, constituyéndose en una ventaja comparativa importante, la cual deberá ser concebida en las estrategias de desarrollo.

Un aspecto a tener en cuenta es la existencia de problemas de conservación de la mayoría de los atractivos, como se muestra en el Figura 4.

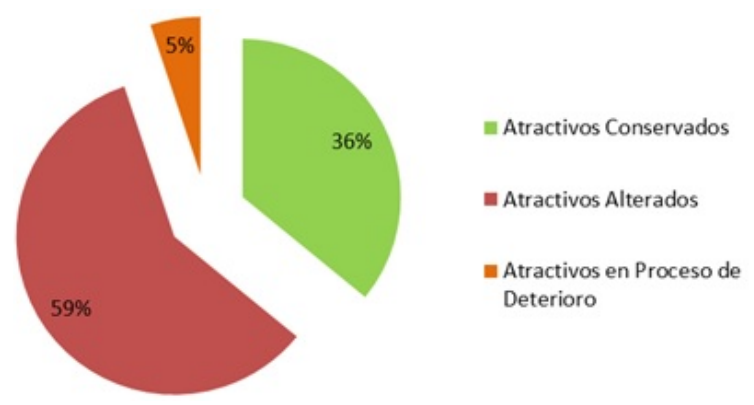

Figura 4. Estado de Conservación de los Atractivo en el Corredor del Santa Bárbara Fuente:Tabla No.1. Elaboración Propia

El $59 \%$ de los atractivos están alterados y el $5 \%$ en proceso de deterioro, lo que hace que se deberá prestar atención a esta situación ya que se puede convertir en una importante debilidad para el territorio en el mediano plazo.

La jerarquización de los atractivos turísticos, se muestra en la Fig. 5. 


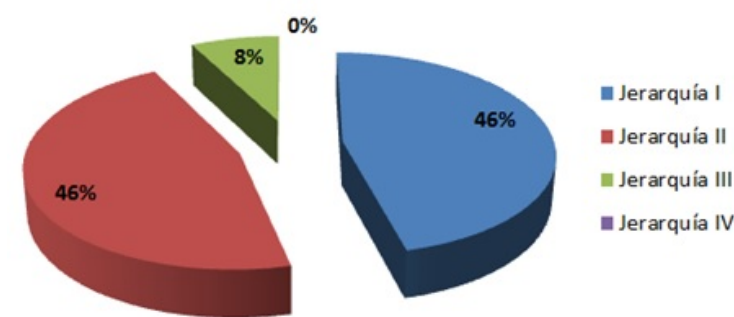

Figura 5. Jerarquización de los Atractivo del Corredor del Santa Bárbara Fuente:Tabla No.1. Elaboración Propia

De los resultados del gráfico se puede interpretar que la mayor parte de los atractivos turísticos del Corredor del Santa Bárbara (el $92 \%$ ) tienen una valoración menor, lo que indica que tienen algún rasgo llamativo, capaz de interesar a visitantes, ya sea de mercado interno y receptivo, que hubiesen llegado a la zona por otras motivaciones turísticas, o de motivar corrientes turísticas actuales o potenciales, y atraer al turismo fronterizo de esparcimiento. También otros sin méritos suficientes para considerarlos a nivel de las jerarquías II, III y IV, pero que igualmente forman parte del patrimonio turístico como elementos que pueden complementar la oferta actual. Esto nos permite comprender la alta potencialidad que tiene el territorio por explotar con fines turísticos dándole valor a aquellos recursos turísticos que todavía se desconocen y que tienen bajos niveles de disfrute por parte del turista.

En relación a la difusión que se realiza de los diferentes atractivos, la Figura 6. muestra que la mayor parte se realiza a nivel local, provincial y nacional.

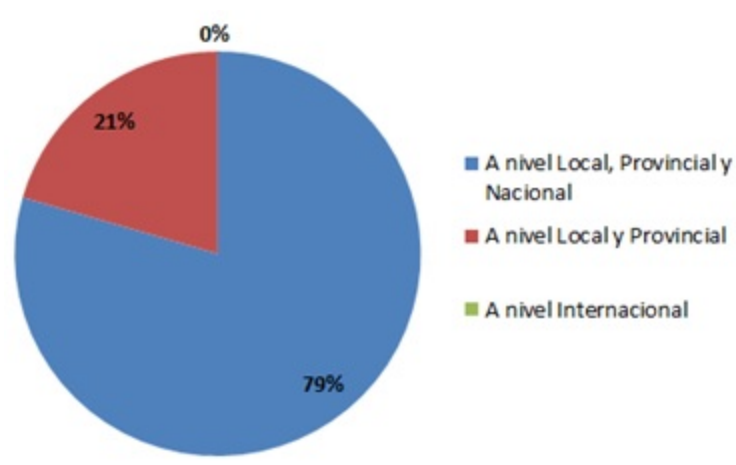

Figura 6. Difusión de los Atractivo del Corredor del Santa Bárbara Fuente:Tabla No.1. Elaboración Propia

También se puede observar que no se hace difusión a nivel internacional lo cual es una debilidad importante si es que se aspira a tener turismo internacional en el territorio. Por lo que se deberían encaminar acciones para estimular la participación en ferias internacionales de turismo, la generación de sitios web que tengan visibilidad internacional, entre otras alternativas.

Otro aspecto que se incorpora y que fundamenta la potencialidad del territorio objeto de estudio es la existencia de una amplia planta turística compuesta por 137 empresas de Alojamiento, Alimentación, Transportación, Recreación y Operación Turística, las cuales fortalecen la imagen del territorio y las posibilidades de avanzar hacia un desarrollo superior de la actividad turística. En la tabla 2 se expone un listado clasificado de las empresas turísticas a las que se hace mención.

Tabla 2 Empresas turísticas por municipio del Corredor del Santa Bárbara

\begin{tabular}{|c|c|c|c|c|}
\hline \multirow{6}{*}{ 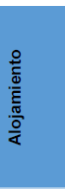 } & CLASIFICACIÓN & GUALACEO & CHORDELEG & SIGSIG \\
\hline & Hosterias & 6 & - & 1 \\
\hline & Hostal & 9 & 2 & - \\
\hline & Hotel & 1 & 1 & \\
\hline & Motel & 1 & - & - \\
\hline & Residencia & 1 & - & 1 \\
\hline \multirow{4}{*}{ 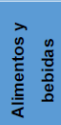 } & Bar & 13 & 1 & 2 \\
\hline & Cafetería & 4 & 1 & 1 \\
\hline & Fuente de soda & 1 & - & - \\
\hline & Restaurants & 38 & 10 & 5 \\
\hline \multirow{2}{*}{ 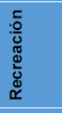 } & Discoteca & 4 & - & - \\
\hline & Termas y Balnearios & 1 & - & - \\
\hline \multirow{2}{*}{ : } & Agencias de viaje & 6 & - & - \\
\hline & Empresas de Transporte & 15 & 5 & 6 \\
\hline \multicolumn{2}{|l|}{ TOTAL } & 101 & 20 & 16 \\
\hline
\end{tabular}

Fuente: Ministerio de Turismo y Jefatura de Turismo de Gualaceo

Como se puede observar existe un predominio de las empresas de alimentación y existe una mayor concentración en el municipio de Gualaceo, lo cual muestra un desbalance en el desarrollo integral del todo el territorio y lo que deberá atenderse en el futuro.

\section{Conclusiones}

Después de haber realizado un análisis de algunos de los elementos del marco teórico existente en la actualidad sobre el turismo y el desarrollo local se puede percibir una tendencia en el primer término a incorporarse los elementos del desarrollo sostenible y en el segundo los aspectos relacionados con el conocimiento y la innovación.

En el caso de Ecuador el turismo se concibe como una alternativa de desarrollo, así se plantea en el PLANDETUR 2020 incrementándose la entrada de extranjeros en el período 2006-2014. Como parte de esta estrategia muchos territorios han emprendido el camino hacia el desarrollo turístico. Este el caso del Corredor del Santa Bárbara, que comprende los municipios de Gualaceo, Chordeleg y SigSig. Este territorio ubicado al sur del país presenta una potencialidad importante para el desarrollo del turismo al contar con 39 atractivos turísticos inventariados. En estos predominan las manifestaciones culturales al contar con varios lugares como Patrimonios de la Nación y de la Humanidad, sitios arqueológicos, gastronomía, fiestas religiosas, artesanía y técnicas milenarias como el IKAT utilizada en la elaboración de las Macanas. También complementa la oferta con varias áreas naturales protegidas y sitios con muy poca intervención humana con mucha potencialidad para el turismo de naturaleza o ecoturismo. 
Aunque se pudo detectar una importante cantidad, el $59 \%$ de los atractivos, que se contabilizan con alteraciones en su conservación se considera que la situación actual del Corredor del Santa Bárbara es un punto de partida para iniciar una estrategia de crecimiento turístico basada en las potencialidades existentes que se constituyen en ventajas comparativas del territorio, a través de las cuales y mediante la implementación de procesos innovadores y de difusión del conocimiento se pueden generar ventajas competitivas que coadyuven al desarrollo del turismo como alternativa de desarrollo local. Si a esto se le agreda la existencia de una extensa planta turística, según la extensión del territorio, se fundamenta la necesidad de los gobiernos locales de emprender acciones que permitan aprovechar la situación actual para impulsar el turismo.

Al ser esta investigación de carácter descriptivo presenta limitaciones como la imposibilidad de establecer causas del desaprovechamiento de los recursos actuales y la propuesta de acciones que cambien la situación descrita. Estas deberán ser consideradas para que próximas investigaciones se encaminen en este sentido.

\section{Referencias Bibliográficas}

Acerenza, M. A. (2011). Sostenibilidad del turismo iii: Confusión sobre la definición del desarrollo sostenible del turismo. Descargado de http:// www. portaldeamerica.com/index.php/ columnistas/miguel-angel-acerenza/ item/5205-sostenibilidad-del

-turismo-iii-confusioC $3 \% B 3 n-s o b r e$

-la-definicioC3B $3 n$-del-desarrollo

-sostenible-del-turismo

Alfaro, E. M. (2007). Continuidades y rupturas entre la actual teoría del desarrollo local y las "viejas"teorias del desarrollo latinoamericano. Reflexiones, 86(2), 9.

Asamblea Constituyente. (2007). Plan estratégico de desarrollo del turismo sostenible para ecuador (PANDETUR 2020). Descargado de http://www.turismo.gob.ec/ wp-content/uploads/downloads/2013/ 02 / P LANDETUR-2020.pdf

Becattini, G. (1979). Dal settore industriale al distretto industrial. alcune conside.

Blacutt, M. (2013). El desarrollo local complementario. un manual para la teoría y la acción. Descargado de http://www. eumed. net/libros -gratis/2013/1252/1252.pdf

Brida, J. G., London, S., y Rojas, M. (2014). El turismo como fuente de crecimiento económico: impacto de las preferencias intertemporales de los agentes. Investigación Económica., LXXIII(289), 59-77.

Brusco, S. (1982). The emilian model: productive decentralisation and social integration. Cambridge journal of economics, 6(2), 167-184.
Castillo, Y., y Lazo, C. (2014). Gestión integrada de desarrollo local sostenible: corredor turístico del santa bárbara, azuay, ecuador (Vol. 17) (n. ${ }^{\circ}$ 4).

Coffey, W. J. and Polese, M. . (1985). Local development: conceptual bases and policy implications. Regional studies, 19(2), 85-93.

Derudder, B., y Witlox, F. (2016). Hub cities in the knowledge economy: seaports, airports, brainports. Routledge.

Dixit, A. K., y Stiglitz, J. E. (1977). Monopolistic competition and optimum product diversity. The American Economic Review, 67(3), 297-308.

Fachinelli, A., y et al. (2014). Capital system, creative economy and knowledge city transformation: insights from bento gonçalves, brazil, expert systems with applications (Vol. 41) (n. ${ }^{\circ}$ 12). Maryland.

Friedmann, M., J.and Douglass. (1978). Agropolitan development: towards a new strategy for regional planning in asia.

Fuà, G. (1983). L'industrializzazione nel nord est e nel centro. industrializzazione senza fratture. Bologna: Il Mulino.

Garofoli, G. (1983). Aree di specializzazione produttiva e piccole imprese in europa. Economia Marche, 2(1).

Helpman, E., y Krugman, P. R. (1985). Market structure and foreign trade: Increasing returns, imperfect competition, and the international economy. MIT press.

Hernández, E. (1991). Planificación turística un enfoque metodológico. México: Trillas.

Hunter, C., Green, H., y cols. (1995). Tourism and the environment: A sustainable relationship? Routledge.

López, D. (1998). Ordenación y planificación integrada de los recursos territoriales turísticos. Castellón-Espa na: Universidad, Jaume.

Mancini, A. (2014, Juli). Dinámicas de desarrollo local en italia: El caso de la iniciativa empresarial femenina en molise. Perfil Coyuntura Económica, No, 23, 3756.

Organización Mundial de Turismo. (2004). National and regional tourism planning. Madrid.

Rodríguez, M. A. (2014). La contribución del turismo al crecimiento económico de méxico: Un análisis por ramas características del sector. Revista Electrónica Nova Scientia, 7(1), 337-351.

Roldán, C. D. (2012). Lineamientos para la planeación del turismo sostenible en el corregimiento de san francisco de asís (municipio de acandí, chocó, colombia). Revista Gestión y Ambiente, 1(15), 129-142.

Rosas, M., y Lara, R. (2013). Desarrollo endógeno local sustentable y propiedad común: San pedro el alto, méxico. Cuadernos de Desarrollo Rural, 10(71), 5980 .

Stöhr, W. B. (1981). Development from below: The bottomup and periphery-inward development paradigm', dalam walter $b$. stohr, development from above or below?: The dialectics of regional planning in deve- 
loping countries. John Wiley and Sons.

Stöhr, W. B. (1985). Industrial structural change and regional development strategies: towards a conceptual framework. Wirtschaftsuniversität Wien, Interdisziplinäres Institut für Raumordnung.

Vázquez, A. (1983). Industrialization in rural areas. the spanish case. (Informe presentado en la Reunión Intergubernamental de la OCDE, celebrada en Senigallia del, 7.)

Vázquez, A. (2000). Documento, primera parte. marco de análisis en enfoque y la política. Descargado de http://cite.flacsoandes.edu.ec/ media/2016/02/Vazquez-A_2000_La

-politica-de-desarrollo-economico

-local.pdf

Vázquez, A. (2007). Desarrollo endógeno. teorías y políticas de desarrollo territorial. Investigaciones
Regionales. Sección Panorama y Debates, 11, 183210.

Recibido: 10 de enero de 2018

Aceptado: 7 de septiembre de 2018 